Structure and Dynamics of Elementary Matter 


\section{NATO Science Series}

\section{A Series presenting the results of scientific meetings supported under the NATO Science Programme.}

The Series is published by IOS Press, Amsterdam, and Kluwer Academic Publishers in conjunction with the NATO Scientific Affairs Division

\section{Sub-Series}

\section{Life and Behavioural Sciences \\ II. Mathematics, Physics and Chemistry \\ III. Computer and Systems Science \\ IV. Earth and Environmental Sciences \\ V. Science and Technology Policy}

\author{
IOS Press \\ Kluwer Academic Publishers \\ IOS Press \\ Kluwer Academic Publishers \\ IOS Press
}

The NATO Science Series continues the series of books published formerly as the NATO ASI Series.

The NATO Science Programme offers support for collaboration in civil science between scientists of countries of the Euro-Atlantic Partnership Council. The types of scientific meeting generally supported are "Advanced Study Institutes" and "Advanced Research Workshops", although other types of meeting are supported from time to time. The NATO Science Series collects together the results of these meetings. The meetings are co-organized bij scientists from NATO countries and scientists from NATO's Partner countries - countries of the CIS and Central and Eastern Europe.

Advanced Study Institutes are high-level tutorial courses offering in-depth study of latest advances in a field.

Advanced Research Workshops are expert meetings aimed at critical assessment of a field, and identification of directions for future action.

As a consequence of the restructuring of the NATO Science Programme in 1999, the NATO Science Series has been re-organised and there are currently Five Sub-series as noted above. Please consult the following web sites for information on previous volumes published in the Series, as well as details of earlier Sub-series.

http://www.nato.int/science

http://www.wkap.nl

http://www.iospress.nl

http://www.wtv-books.de/nato-pco.htm

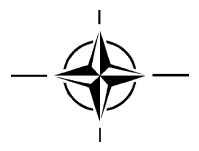

Series II: Mathematics, Physics and Chemistry -Vol. 166 


\title{
Structure and Dynamics of Elementary Matter
}

edited by

\author{
Walter Greiner \\ Johann Wolfgang Goethe University, \\ Institute for Theoretical Physics, \\ Frankfurt, Germany
}

\section{Mikhail G. Itkis}

Joint Institute of Nuclear Research,

Dubna, Russia

\section{Joachim Reinhardt}

Johann Wolfgang Goethe University, Institute for Theoretical Physics,

Frankfurt, Germany

and

\section{Mehmet Cem Güçlü}

Istanbul Technical University,

Turkey

Springer Science+Business Media, B.V. 
Proceedings of the NATO Advanced Study Institute on

Structure and Dynamics of Elementary Matter

Camyuva-Kemer (Antalya), Turkey

22 September-2 October 2003

A C.I.P. Catalogue record for this book is available from the Library of Congress.

ISBN 978-1-4020-2446-7

ISBN 978-1-4020-2705-5 (eBook)

DOI 10.1007/978-1-4020-2705-5

Printed on acid-free paper

All Rights Reserved

(C) 2004 Springer Science+Business Media Dordrecht

Originally published by Kluwer Academic Publishers in 2004

Softcover reprint of the hardcover 1st edition 2004

No part of this work may be reproduced, stored in a retrieval system, or transmitted in any form or by any means, electronic, mechanical, photocopying, microfilming, recording or otherwise, without written permission from the Publisher, with the exception of any material supplied specifically for the purpose of being entered and executed on a computer system, for exclusive use by the purchaser of the work. 


\section{TABLE OF CONTENTS}

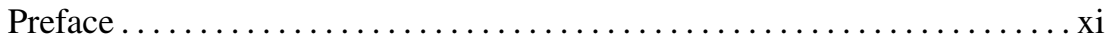

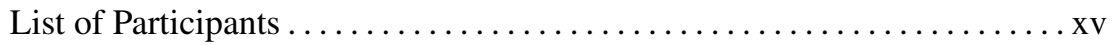

\section{Relativistic Heavy Ion Physics - Experiment}

J.W. Harris:

Creating Bulk QCD Matter at RHIC .....................

R. Stock:

The Strangeness Signal in Hadron Production at Relativistic Energy . . . 19

J. Baudot:

Multistrange Baryons at STAR $\ldots \ldots \ldots \ldots \ldots \ldots \ldots \ldots \ldots \ldots \ldots \ldots \ldots \ldots \ldots \ldots \ldots \ldots \ldots \ldots$

C. Blume for the NA49 collaboration:

Recent Results from the NA49 Experiment .................... 53

D. d'Enterria for the PHENIX collaboration:

QCD Hard Scattering Results from PHENIX at RHIC .............61

S.V. Greene:

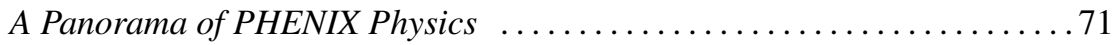

H.H. Gutbrod:

The Future International Accelerator Facility for Beams of Ions and

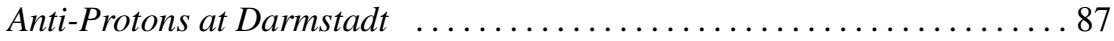

C. Kuhn for the ALICE collaboration:

Strangeness Production and Prospects for $L H C \ldots \ldots \ldots . \ldots 5$

C. Markert for the STAR collaboration:

Resonance Production in Relativistic Heavy Ion Collisions . . . . . . . . . 103

W. Reisdorf:

Constraints on the Nuclear EOS from Heavy Ion Data in the SIS

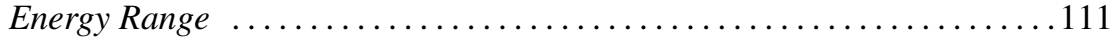

G.D. Westfall for the STAR collaboration:

Balance Functions at RHIC 


\section{Relativistic Heavy Ion Physics - Theory}

L.P. Csernai, Andrea Anderlik, Cs. Anderlik, A. Keranen, V.K. Magas, J. Manninen, E. Molnár, Ágnes Nyíri, B.R. Schlei, D.D. Strottman and K. Tamousiunas:

Phase Transitions in High Energy Heavy-Ion Collisions

C. Greiner, S. Juchem, Z. Xu:

Aspects of Non-Equilbrium Quantum Field Theory in Relativistic Heavy Ion Collisions

M. Gyulassy:

The QGP Discovered at RHIC

L. McLerran:

What is the Evidence for the Color Glass Condensate?

I.N. Mishustin:

Clusters of Matter and Antimatter

S.A. Bass, R.J. Fries, C. Nonaka:

Hadronization at RHIC: Interplay of Recombination and Fragmentation 225

E.L. Bratkovskaya, M. Bleicher, A. Dumitru, K. Paech, M. Reiter, S. Soff, H. Stöcker, H. Weber, M. van Leeuwen, W. Cassing:

Review of QGP Signatures - Ideas versus Observables

F. Cerutti, F. Ballarini, G. Battistoni, M. Cavinato, A. Empl, E. Fabrici, A. Fassò, A. Ferrari, E. Gadioli, E. Gadioli Erba, M.V. Garzelli, A. Ottolenghi, V. Parini, L.S. Pinsky, J. Ranft, P. R. Sala:

Towards a Comprehensive Description of Heavy Ion Reactions ...... 255

M. Gorenstein:

Signals of Deconfinement Transition in Nucleus-Nucleus Collisions . ...265

\section{Astroparticle and Nuclear Astrophysics}

T. Bollenbach, T. Strother, W. Bauer:

3D Core-Collapse Supernova Calculations

K. Pretzl:

Dark Matter, Massive Neutrinos and SUSY Particles

J. Schaffner-Bielich:

Structure of Stars and Nuclei

A. Schmitt:

Spin-One Color Superconductivity in Neutron Stars 
I. Shovkovy, M. Huang:

Theory of Gapless Superconductivity in Quark Matter

\section{Superheavy Elements}

S. Hofmann:

Exotic Nuclei - The Experimental Quest

Yu.Ts. Oganessian:

Experimental Search for Superheavy Nuclei

A. Sobiczewski, I. Muntian, O. Parkhomenko:

Description of Properties of Superheavy Nuclei

Y. Abe, B. Bouriquet, G. Kosenko:

Dissipation-Fluctuation Dynamical Approach to Fusion leading to the

Superheavy Elements

Y. Aritomo, M. Ohta, T. Materna, F. Hanappe, L. Stuttgé:

Fusion-Fission Dynamics of Superheavy Nuclei by the Fluctuation-Dissipation Model

T. J. Bürvenich, P.-G. Reinhard, J. A. Maruhn, W. Greiner :

Self-Consistent Mean-Field Models and their Application to Superheavy

Nuclei

R.A. Gherghescu, W. Greiner:

Influence of Deformation on the Synthesis of Superheavy Elements

M.G. Itkis:

Shell Effects in Fission and Quasi-Fission of Heavy and

Superheavy Nuclei

I. Muntian, A. Sobiczewski:

Fission Barriers of Heavy and Superheavy Nuclei

\section{Exotic Nuclei and Nuclear Structure}

G.G. Adamian, N.V. Antonenko, S.P. Ivanova, W. Scheid, A. Zubov: Fusion and Quasifission in a Molecular Model

J.S. Vaagen, G. Hagen, B.V. Danilin, S.N. Ershov, I.J. Thompson:

Exotic Matter at the Low Density Limit: Exploring Bound and Continuum Structures of Borromean Halo Nuclei

A. Diaz-Torres:

Formation of the Compound Nucleus in Fusion of Heavy Nuclei 
viii

D.S. Delion, A. Insolia, R. J. Liotta

Anisotropic $\alpha$ - Decay

S. Galès:

Exotic Nuclei: Where do we go!

G. Münzenberg:

Storage-Ring Experiments with Exotic Nuclei: from Mass Measurements to the Future

D.N. Poenaru, W. Greiner:

New Candidates for Cluster Decay Experiments

A.V. Ramayya, J.H. Hamilton, J.K. Hwang, P. Gore, E.F. Jones, D. Fong, G.M. Ter Akopian, A.V. Daniel, J.O. Rasmussen, S.-C. Wu, R. Donangelo, J. Kormicki, X.Q. Zhang, A. Rodin, A. Formichev, J. Kliman, L. Krupa, G. Chubaryan, J.D. Cole:

Nuclear Fission and Structure Studies with Gammasphere

P. Ring:

Relativistic Density Functional Theory: Applications to Nuclei far from

Stability

S. Schramm:

Nuclei and Nuclear Matter in a Hadronic Model

G.M. Ter-Akopian, A.V. Daniel, A.S. Fomichev, G.S. Popeko, A.M. Rodin, J.H. Hamilton, A.V. Ramayya, J. Kormicki, J.K. Hwang, D. Fong, P. Gore, J.O. Rasmussen, I.Y. Lee, A.O. Macchiavelli, P. Fallon, J.D. Cole, W. Greiner: New Experimental Approach to Ternary Fission Relying on the $\gamma$-Ray

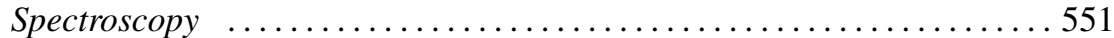

A.S. Umar, V.E. Oberacker, E. Teran, A. Blazkiewicz:

HFB Calculations for Nuclei far from Stability

\section{Miscellaneous}

T. S. Biró, G. Purcsel, B. Müller:

How low is the Thermodynamical Limit?

S. Hossenfelder, M. Bleicher and H. Stöcker:

Signatures of Large Extra Dimensions

C. Keil, H. Lenske, C. Greiner:

Baryon-Baryon Interactions in Free Space and Matter

H. Kleinert:

No Pions in Chiral Nambu-Jona-Lasinio Model with Less than Three Quark Colors 
J. Ruppert, D. Röder:

Self-Consistent Description of Strongly Interacting Matter at High

Temperatures

A.V. Solov'yov:

The Physics of Clusters

\section{Posters}

N.A.Enaki, V.V. Eremeev:

Phase Transition in Superconductivity at Two-Phonon Exchange Between the Electrons

K.A. Gridnev, K.V. Ershov, V.G. Kartavenko, W. Greiner:

Factorization of Charge and Nuclear Formfactors

K. Karimov, R. Gainutdinova:

Environmental Contaminations of Mountain Regions: Problems and Decisions 645

I.P. Lokhtin, S.V. Petrushanko, L.I. Sarycheva, A.M. Snigirev:

Using Calorimetry to Investigate the Azimuthal Anisotropy in

Heavy-Ion Collisions

I.P. Lokhtin, A.M. Snigirev:

Jet Quenching Studies in Heavy Ion Collisions at LHC

V.K. Magas, H. Satz:

Deconfinement and Freeze-Out Conditions for Hadronic Matter

N. Paar, T. Nikšić, D. Vretenar, P. Ring:

Relativistic QRPA Description of Excitations in Exotic Nuclei

S. Salur for the STAR Collaboration:

$\Sigma(1385)$ Resonance Studies with STAR at $\sqrt{s_{N N}}=200 \mathrm{GeV}$

N. Türkan, H. Erdem, D. Olgun, İ. Uluer, S. İnan:

The Investigation of Multipolarity of the Electromagnetic Transitions in some even-even Hf Isotopes

M.B. Yucel, N. Unal:

Quantum Dynamics of Brachistochrone Problem 


\section{PREFACE}

Ladies and gentlemen,

dear colleagues, welcome to Kemer to the NATO Advanced Study Institute

Structure and Dynamics of Elementary Matter.

We have chosen Kemer as the place of our NASI because it is located in a beautiful and hospitable surrounding. This part of the Mediterranean at the Turkish Riviera is a historic region where many cultures meet (e.g., the Oriental and the Greek and Roman European cultures) and where you find numerous places which played a role in ancient science and in early Christianity. Moreover, with the hotel Ceylan Inter-Continental we have found a most excellent meeting place, directly located at the beach, equipped with wonderful swimming pools and restaurants - an absolutely first-class location.

Our NASI will deal with the most recent developments in high-energy heavy ion physics and in the search for superheavy nuclei - two rather distinct areas of research. Indeed, we want to bring two very active communities of nuclear and high-energy physics into close contact. The meeting is both a school and has also the character of a conference: A school because there are many advanced students, many of which are themselves already top researchers and who are contributing with their own research in seminars and posters. It is also a conference because new results in the exciting and wonderful fields of low- and high-energy heavy ion physics will be presented. We are mainly focussing on the topics of superheavy elements and of hot and dense nuclear matter.

Here we investigate, how elementary matter may have been formed and what its properties were just fractions of a second after the big bang. We try to reveal how our world was formed, how baryosynthesis and nucleosynthesis may have proceeded, how ordinary or exotic stars (e.g., neutron or quark stars) are built, how supernova explosions occur, and many similar questions. It is believed that hot and/or dense elementary matter displays completely new phases, most notably the famous Quark-Gluon-Plasma predicted by Quantum Chromodynamics (QCD) which most probably is a cluster plasma where strong correlations of hadronic type survive. The most recent experimental data from RHIC 
indicate that we are close to the discovery of this new phase of elementary matter. Another intriguing phenomenon are the phases of Color Superconductivity which may exist in the interior of collapsed stars. Other new forms of matter involving strangeness (strangelets, multi- $\Lambda$-hypernuclei, multiply strange exotic mesonic objects (Memos)) may be formed in nuclear collisions, as well as clusters of matter and antimatter. The extension of our periodic system into the new directions of strange matter and antimatter leads to exciting prospects. The matter-antimatter clusters, furthermore, open the door for a new form of cold compression of elementary matter. This would stand in contrast to the compression by nuclear shock waves which automatically also heats up the matter (Rankine-Hugoniot equations). We want to create dense nuclear matter and this up to now was achieved through the shock wave mechanism in its various forms. When nuclei collide at high energies they compress and heat up tremendously. On the other hand, in matter-antimatter clusters the heating should be much less (Mishustin, Satarov, ...).

Another tremendously rich and exciting field is the search for superheavy nuclei. The theoretical predictions by the Lund and Frankfurt groups now are 35 years old. Experiments at GSI (S. Hofmann, Münzenberg, and collaborators) and at Dubna (Oganessian and his group) have brought great progress. Shortliving superheavy nuclei along the proton drip line were found and their $\alpha$ decay has been observed. The fusion of these superheavy nuclei discovered so far occurs if the projectile and target are chosen such that they approach through a cold valley in the potential energy surface. In this case no push and also no 'extra push' is necessary. The fused elements are produced rather cold.

Also quasifission processes (Itkis) of various nuclear systems are seen. The theory has been better founded over the years, using, e.g., the framework of meson field theory, and new magic numbers occur. The extension of the periodic system is a challenge!

We want to bring both research communities together. It is important to understand each other, to talk to each other and to learn from each other! This is why in this NASI we have mixed the talks evenly, so that there is little possibility to escape each other.

We will have plenty of discussions, I hope, both here in the lecture hall as well as outside in the surroundings and at the beach side. The wonderful hotel Ceylan will provide an ideal setting for our conference and we will spend nearly two weeks in a most agreeable confinement.

As it turned out, we had far too many applications, many of which had to be turned down, and also the number of accepted participants lies somewhat above the normal quota for a NATO Advanced Studies Institute. Not surprisingly, quite a number of spouses and companions have joined their husbands and friends.

Let me finally make a number of remarks concerning the organization of the meeting. 
The idea for such a NASI down here in Turkey has been suggested to me by A. V. Ramayya (Vanderbilt University) and I took it up with pleasure. My colleagues and friends Dr. Joachim Reinhardt from Goethe University, Frankfurt, and Prof. Çem Güçlü from Istanbul Technical University were helping tremendously in the organization. I thank them cordially. NATO's scientific program has been very generous in providing the main support for this school, deserving our warmest thanks. The German Ministry for Science (BMBF), in particular Dr. D. Müller, and the Gesellschaft für Schwerionenforschung (GSI, Prof. Walter Henning) have also been very helpful with their support. The same is true for the Institut National de Physique Nucléaires et de Physique Particules (IN2P3), for Vanderbilt University, Nashville TN (Prof. J. Hamilton) and for the Turkish Atomic Energy Agency, Ankara. All of them deserve our "thank you" for their support, without which this meeting could not take place in this form. Travel support for students was granted by the Scientific and Technical Research Council of Turkey (Tübitak) and by the National Science Foundation, Washington.

Special acknowledgments go once more to Dr. Joachim Reinhardt who keeps contact with all the speakers and will be organizing most of the editorial work for the Proceedings volume.

The next eleven days will be time filled with hard work, but I am confident that it will also be a fruitful, memorable, and enjoyable period.

I wish us all a very pleasant, interesting and successful NASI. Let us get to work now!

Walter Greiner 


\section{NATO ADVANCED STUDIES INSTITUTE}

\section{"Structure and Dynamics of Elementary Matter"}

Kemer/Turkey, Sept. 22 - Oct. 2,2003

\section{List of Participants}

\section{Abe, Yasuhisa}

Yukawa Institute for Theoretical Physics

Kyoto University

Kyoto 606-8502

Japan

abey@yukawa.kyoto-u.ac.jp

\author{
Aichelin, Jörg \\ SUBATECH \\ Ecole des Mines, B.P. 20722 \\ 4, Rue Alfred Kastler \\ F-44307 Nantes, Cedex 03 \\ France \\ Aichelin@subatech.in2p3.fr
}

\section{Aritomo, Yoshihiro}

Flerov Lab of Nuclear Reactions

Joint Institute of Nuclear Research

141908 Dubna,

Moscow Region, Russia

Aritomo@nt.phys.s.u-tokyo.ac.jp

Bass, Steffen

Duke University

Department of Physics

Physics Bldg., Science Dr.

Box 90305

Durham, NC 27708

USA

bass@phy.duke.edu

\section{Baudot, Jerome}

Institut de Recherches Subatomiques (IReS)

23, rue du Loess

BP 28

67037 Strasbourg Cedex 2

France

baudot@in2p3.fr

\section{Bauer, Wolfgang}

Department of Physics and Astronomy

Michigan State University

East Lansing, MI 48824-1116

USA

Bauer@pa.msu.edu

\section{Bezverkhny, Betty}

Yale University

A.W. Wright Nuclear Structure Laboratory

272 Whitney Avenue

New Haven, CT 06520-8124

USA betty.bezverkhny@yale.edu

\section{Biró, Tamas}

Theoretical Department

Research Institute for Particle and Nuclear

Physics

P.O.B. 49

1525 Budapest

Hungary

tsbiro@sunserv.kfki.hu

Blume, Christoph

Institut für Kernphysik

Johann Wolfgang Goethe-Universität

August-Euler-Straße 6

60486 Frankfurt

Germany

blume@ikf.uni-frankfurt.de

\section{Bürvenich, Thomas}

Los Alamos National Laboratory

T16, MS B283

Los Alamos NM 87545

USA

tbuerven@lanl.gov

\section{Büyükcizmeci, Nihal}

Department of Physics

Faculty of Science and Arts

Selcuk University

42079, Konya

Turkey

nihal@selcuk.edu.tr

\section{Catu, Oana}

Yale University

New Haven CT

USA

oana.catu@yale.edu

\section{Cerutti, Francesco}

Universita' degli Studi di Milano

Dipartimento di Fisica

via Celoria, 16

20133 Milano

Italy

Francesco.Cerutti@mi.infn.it

\section{Coffin, Jean Pierre}

Institut de Recherches Subatomiques

23 Rue du Loess BP28

F 67037 Strasbourg Cedex 2 
France

coffin@in2p3.fr

Csernai, Laszlo P.

Section for Theoretical and Computational Physics

University of Bergen

Allegaten 55

N 5007 Bergen

Norway

csernai@fi.uib.no

d'Enterria, David G.

Nevis Laboratories

Columbia University, NY

and

Brookhaven National Laboratory

Phys. Dept. Bldg 510C

Upton, 11973, NY

USA

denterria@nevis.columbia.edu

Diaz-Torres, Alexis

Institut für Theoretische Physik

Johann Wolfgang Goethe-Universität

Robert Mayer Str. 10

60054 Frankfurt am Main

Germany

diaz@th.physik.uni-frankfurt.de

\section{Dikmen, Erdal}

Suleyman Demirel Universitesi

Fen-Edebiyat Fakultesi, Fizik Bolumu Isparta

Turkey

and

Drexel University

Physics Department

Philadelphia,PA, 19151

USA

erdalsdikmen@yahoo.com

Eremeev, Vitalie

Institute of Applied Physics,

Academy of Sciences of Moldova

Academiei str 5

Chisinau MD-2028

Moldova

eremeev@cc.acad.md

\section{Eren, Nuretdin}

Selcuk University

Arts and Science Faculty

Department of Physics

42079 Kampus/Konya

Turkey

eren@selcuk.edu.tr

Fong, Dennis

Department of Physics and Astronomy
Vanderbilt University

Nashville, TN 37235

USA

d.fong@vanderbilt.edu

\author{
Gainutdinova, Razia \\ Institute of Physics \\ National Academy of Sciences \\ Chui Prosp., 265-A \\ Bishkek 720071 \\ Kyrgyz Republic \\ epfk@rambler.ru
}

\section{Gales, Sydney}

Institute de Physique Nucléaire

15 , rue Georges Clémenceau

F-91406 Orsay cedex

France

gales@ipno.in2p3.fr

\section{Gelir, Ali}

Istanbul Technical University

Fizik Bolumu, Ayazaga kampusu

Maslak

Istanbul

Turkey

gelira@itu.edu.tr

\section{Gherghescu, Radu}

Horia Hulubei National Institute of Physics and Nuclear Engineering

Str. Atomistilor 407

P. O. Box MG-6

RO-76900 Bucharest-Magurele

Romania

rgherg@ifin.nipne.ro

\section{Gorenstein, Mark}

Bogolyubov Institute for Theoretical Physics 14b Metrologichna str.

$252143 \mathrm{Kiev}$

Ukraine

mark@mgor.kiev.ua

Greene, Victoria

Department of Physics and Astronomy

Vanderbilt University

Box 1807, Station B

Nashville, TN 37235

USA

senta.v.greene@vanderbilt.edu

\section{Greiner, Carsten}

Institut für Theoretische Physik

Justus-Liebig-Universität

Heinrich-Buff-Ring 16

35392 Giessen

Germany

Carsten.Greiner@theo.physik.uni-giessen.de 


\section{Greiner, Walter}

Institut für Theoretische Physik

Johann Wolfgang Goethe-Universität

Robert Mayer Str. 10

60054 Frankfurt am Main

Germany

Greiner@th.physik.uni-frankfurt.de

\section{Gridnev, Konstantin}

St. Petersburg State University

Institute of Physics

Uljanova 1, Peterhof

St.Petersburg, 198504

Russia

gridnev@nuclpc1.phys.spbu.ru

\section{Güçlï, Cem}

Istanbul Technical University

Department of Physics

Maslak 80626 Istanbul

Turkey

guclu@itu.edu.tr

\section{Gutbrod, Hans}

Gesellschaft für Schwerionenforschung

Postfach 110552

64220 Darmstadt

Germany

H.Gutbrod@gsi.de

\section{Gyulassy, Miklos}

Columbia University

Physics Department

538 West 120th Street

New York, NY 10027

USA

gyulassy@nt3.phys.columbia.edu

\section{Hagen, Gaute}

Fysisk institutt

Universitetet i Bergen

Allégt. 55

5007 Bergen

Norway

Gaute.Hagen@fi.uib.no

\section{Harris, John}

Physics Department

Wright Nuclear Structure Laboratory

Yale University

272 Whitney Avenue

New Haven, CT 06520-8124

USA

John.Harris@yale.edu

\section{Hofmann, Sigurd}

Gesellschaft für Schwerionenforschung

Postfach 110552

64220 Darmstadt

Germany
S.Hofmann@gsi.de

Holmes, Martha

Department of Physics and Astronomy

Vanderbilt University

Box 1807, Station B

Nashville, TN 37235

USA

martha.j.holmes@vanderbilt.edu

Hossenfelder, Sabine

Institut für Theoretische Physik

Johann Wolfgang Goethe-Universität

Robert Mayer Str. 10

60054 Frankfurt am Main

Germany

hossi@th.physik.uni-frankfurt.de

\section{Hostler, Jacquelyn \\ Berea College \\ Department of Physics \\ CPO 2191 \\ Berea, KY 40404 \\ USA \\ Jaki_Hostler@berea.edu}

Insolia, Antonio

Dipartimento di Fisica e Astronomia \& INFN

Universita`di Catania

Via S. Sofia, 64

I-95123 Catania

Italy

Antonio.Insolia@ct.infn.it

Itkis, Mikhail

Flerov Laboratory of Nuclear Reactions

Joint Institute for Nuclear Research

141980 Dubna, Moscow region

Russian Federation

itkis@flnr.jinr.ru

Karimov, Kazimir

Institute of Physics

National Academy of Sciences

Chui Prosp., 265-A

Bishkek 720071

Kyrgyz Republic

kazimir@aknet.kg

Keil, Christoph

Institut für Theoretische Physik

Justus-Liebig-Universität

Heinrich-Buff-Ring 16

35392 Giessen

Germany

christoph.m.keil@theo.physik.uni-giessen.de

Kezzar, Khalid

Gesellschaft für Schwerionenforschung

Postfach 110552 
64220 Darmstadt

Germany

k.kezzar@gsi.de

\section{Kleinert, Hagen}

Institut für Theoretische Physik

Freie Universität Berlin

Arnimallee 14

14195 Berlin

Germany

Hagen.kleinert@physik.fu-berlin.de

\section{Kostyuk, Andriy}

Bogolyubov Institute for Theoretical Physics

$14 \mathrm{~b}$ Metrologichna str.

$252143 \mathrm{Kiey}$

Ukraine

kostyuk@th.physik.uni-frankfurt.de

Kozlov, Michael

HEP department

School of Physics

Tel Aviv University

Ramat Aviv, Tel Aviv 69978

Israel

bmk007@motorola.com

\section{Kuhn, Christian}

Institut de Recherches Subatomiques

23 Rue du Loess - BP 28

67037 Strasbourg Cedex 2

France

Christian.kuhn@ires.in2p3.fr

\section{Lokhtin, Igor}

D.V. Skobeltsyn Institute of Nuclear Physics

Moscow State University

119992, Vorobievy Gory

Moscow

Russia

igor@lav01.sinp.msu.ru

\section{Ludolphs, Wilrid}

Physikalisches Institut

Philosophenweg 12

69120 Heidelberg

Germany

ludolphs@physi.uni-heidelberg.de

\section{Magas, Volodymyr}

Center for Physics of Fundamental Interactions (CFIF)

Instituto Superior Tecnico

Av. Rovisco Pais,

1049-001 Lisboa,

Portugal

vladimir@cfif.ist.utl.pt

\section{Markert, Christina}

Physics Department
Wright Nuclear Structure Laboratory

Yale University

272 Whitney Avenue

New Haven, CT 06520-8124

USA

christina.markert@yale.edu

\section{McLerran, Larry}

Brookhaven National Laboratory

Physics Department/Nuclear Theory Group

P.O.Box 5000

Upton, NY 11973-5000

USA

mclerran@bnl.gov

Mishustin, Igor

Russian Research Center

Kurchatov Institute

Kurchatov sq. 1

Moscow, 123182

Russia

mish@mbslab.kiae.ru

\section{Mişicu, Serban}

Department of Theoretical Physics

Institute of Atomic Physics

Magurele-Bucharest, MG-6

Romania

misicu@theorl.theory.nipne.ro

\section{Molnar, Etele}

University of Bergen

Department of Physics

Allegaten 55

5075 Bergen

Norway

etele@ffi.uib.no

\section{Muntian, Igor}

Soltan Institute for Nuclear Studies,

Hoza 69

PL-00-681 Warsaw

Poland

muntian@fuw.edu.pl

\section{Münzenberg, Gottfried}

Gesellschaft für Schwerionenforschung

Postfach 110552

64220 Darmstadt

Germany

G.Muenzenberg@gsi.de

\section{Oganessian, Yuri}

Flerov Laboratory of Nuclear Reactions

Joint Institute for Nuclear Research

141980 Dubna, Moscow region

Russia

oganessian@sungraph.jinr.ru

\section{Ogul, Riza}


Selcuk University

Department of Physics

42079 Kampus

Konya

Turkey

rogul@selcuk.edu.tr

\section{Onbasli, Ulker}

University of Marmara

Department of Physics

Ridvanpasa cad. 2. sok. $85 / 12$

Göztepe/Istanbul

Turkey

\section{Özcan, Aynur}

Gazi University

Physics Department

06500 Teknikokullar

Ankara

Turkey

aynur@gazi.edu.tr

\section{Özel, Banu}

Department of Physics

Faculty of Arts and Sciences

Cukurova University

01330, Adana

Turkey

banuozel@cu.edu.tr

\section{Paar, Nils}

Physik-Department T30

Technische Universität München

James-Franck-Strasse 1

85748 Garching

Germany

npaar@ph.tum.de

\section{Parkhomenko, Alexandr}

Soltan Institute for Nuclear Studies,

Hoza 69

PL-00-681 Warsaw

Poland

shura@fuw.edu.pl

\section{Petrushanko, Sergey}

Hadronic Interactions Laboratory,

Skobeltsyn Institute of Nuclear Physics,

Moscow State University,

Moscow 119992

Russia

sergant@lav01.sinp.msu.ru

\section{Plostinar, Dan-Ciprian}

Babes-Bolyai University

Romania

pcipri@yahoo.com

\section{Poenaru, Dorin}

Horia Hulubei National Institute of Physics and Nuclear Engineering

Str. Atomistilor 407

P. O. Box MG-6

RO-76900 Bucharest-Magurele

Romania

poenaru@ifin.nipne.ro

\section{Pretzl, Klaus}

Laboratorium für Hochenergiephysik

Universität Bern

Sidlerstr. 5

CH 3012 Bern

Schweiz

Klaus.Pretzl@lhep.unibe.ch

Ramayya, Akunuri V.

Department of Physics and Astronomy

Vanderbilt University

VU Station B 1807

Nashville, TN 37235

USA

a.v.ramayya@vanderbilt.edu

Rami, Fouad

Institut de Recherches Subatomiques - IRES

23 Rue du Loess - BP 28

67037 Strasbourg Cedex 2

France

fouad.rami@ires.in2p3.fr

Reinhardt, Joachim

Institut für Theoretische Physik

Johann Wolfgang Goethe-Universität

Robert Mayer Str. 10

60054 Frankfurt am Main

Germany

jr@th.physik.uni-frankfurt.de

Reisdorf, Willibrord

Gesellschaft für Schwerionenforschung

Postfach 110552

64220 Darmstadt

Germany

W.Reisdorf@gsi.de

\section{Renfordt, Rainer}

Institut für Kernphysik

Johann Wolfgang Goethe-Universität

August-Euler-Straße 6

60486 Frankfurt

Germany

renfordt@ikf.uni-frankfurt.de

\section{Reuter, Philipp}

Institut für Theoretische Physik

Johann Wolfgang Goethe-Universität

Robert Mayer Str. 10

60054 Frankfurt am Main

Germany 
preuter@th.physik.uni-frankfurt.de

\section{Ring, Peter}

Physik-Department T30

Technische Universität München

James-Franck-Strasse 1

85748 Garching

Germany

Ring@ph.tum.de

\section{Rischke, Dirk}

Institut für Theoretische Physik

Johann Wolfgang Goethe-Universität

Robert Mayer Str. 10

60054 Frankfurt am Main

Germany

drischke@th.physik.uni-frankfurt.de

\section{Ruppert, Jörg}

Institut für Theoretische Physik

Johann Wolfgang Goethe-Universität

Robert Mayer Str. 10

60054 Frankfurt am Main

Germany

ruppert@th.physik.uni-frankfurt.de

\section{Sa'd, Basil}

Physics Dept.

Yarmouk University

Irbid 21163

Jordan

basil.sad@mail.com

\section{Salomov, Uktam}

Heat Physics Department

of the Uzbek Academy of Sciences

28 Katartal St.

700135, Tashkent

Uzbekistan

lps@mnet.uz

\section{Salur, Sevil}

Yale University

POB 204285

New Haven, CT, 06520

USA

sevil.salur@yale.edu

\section{Schaffner-Bielich, Jürgen}

Institut fuir Theoretische Physik

Johann Wolfgang Goethe-Universität

Robert Mayer Str. 10

60054 Frankfurt am Main

Germany

schaffner@th.physik.uni-frankfurt.de

\section{Scheid, Werner}

Institut für Theoretische Physik

Justus-Liebig-Universität

Heinrich-Buff-Ring 16

\section{Giessen}

Germany

werner.scheid@theo.physik.uni-giessen.de

\section{Schmitt, Andreas}

Institut für Theoretische Physik

Johann Wolfgang Goethe-Universität

Robert Mayer Str. 10

60054 Frankfurt am Main

Germany

aschmitt@th.physik.uni-frankfurt.de

\section{Schramm, Stefan}

Institut für Theoretische Physik

Johann Wolfgang Goethe-Universität

Robert Mayer Str. 10

60054 Frankfurt am Main

Germany

schramm@th.physik.uni-frankfurt.de

\section{Shovkovy, Igor}

Institut für Theoretische Physik

Johann Wolfgang Goethe-Universität

Robert Mayer Str. 10

60054 Frankfurt am Main

Germany

shovkovy@th.physik.uni-frankfurt.de

\section{Sobiczewski, Adam}

Soltan Institute for Nuclear Studies,

Hoza 69

PL-00-681 Warsaw

Poland

Adam.Sobiczewski@fuw.edu.pl

\section{Solov'yov, Andrey}

A.F. Ioffe Physical Technical Institute

Russian Academy of Sciences

Polytechnicheskaja str. 26

St. Petersburg 194021

Russia

E-mail: solovyov@rpro.ioffe.rssi.ru

\section{Solov'yov, Ilya}

A.F. Ioffe Physical Technical Institute Russian Academy of Sciences

Polytechnicheskaja str. 26

St. Petersburg 194021

Russia

E-mail: ilia@th.physik.uni-frankfurt.de

Stock, Reinhard

Institut für Kernphysik

Johann Wolfgang Goethe-Universität

August-Euler-Straße 6

60486 Frankfurt

Germany

stock@ikf.uni-frankfurt.de 
Institut für Theoretische Physik Johann Wolfgang Goethe-Universität Robert Mayer Str. 10

60054 Frankfurt am Main

Germany

stoecker@th.physik.uni-frankfurt.de

\section{Strottman, Dan}

Theoretical Division

MS B210

Los Alamos National Laboratory

Los Alamos, NM 87545

USA

dds@lanl.gov

Sucu, Yusuf

Akdeniz University

Faculty of Sciences and Arts

Department of Physics

P.B.510, 07058, Antalya

Turkey

\section{Svirikin, Alexander}

Flerov Laboratory of Nuclear Reactions Joint Institute for Nuclear Research Joliot-Curie 6

141980 Dubna, Moscow region

Russia

sasha@sunvas.jinr.ru

\section{Ter-Akopian, Gurgen}

Joint Institute for Nuclear Research 141980 Dubna

Russia

Gurgen.TerAkopian@jinr.ru

\section{Türkan, Nurettin}

Erciyes University

Yozgat Fenedebiyat Fakültesi

66100 Yozgat

Turkey

nurettin_turkan@yahoo.com

\section{Turkoz, Semsettin}

Ankara University

Department of Physics

06100 Tandogan Ankara

Turkey

turkoz@science.ankara.edu.tr

\section{Udovyk, Oleg}

ITP, Ukrainian Academy of Sciences

3, Dobrohotov St.

Kyiv 03142

Ukraine

oleg_udovyk@hotmail.com

\section{Umar, Sait}

Department of Physics and Astronomy Vanderbilt University
Box 1807, Station B

Nashville, TN 37235

USA

Umar@compsci.cas.vanderbilt.edu

Ünal, Nuri

Akdeniz University

Faculty of Sciences and Arts

Department of Physics

P.B.510, 07058, Antalya

Turkey

nuriunal@akdeniz.edu.tr

Vaagen, Jan

Fysisk institutt

Universitetet i Bergen

Allégt. 55

5007 Bergen

Norway

JanS.Vaagen@fi.uib.no

\section{Voinov, Alexei}

Flerov Laboratory of Nuclear Reactions Joint Institute for Nuclear Research

Joliot-Curie St., 6

Dubna 141980

Russia

voinov2000@mail.ru

\section{Westfall, Gary}

National Superconducting Cyclotron

Laboratory

Department of Physics and Astronomy

Michigan State University

East Lansing, Michigan 48824-1321

USA

Westfall@nscl.msu.edu

\section{Wheaton, Spencer}

University of Cape Town

Department of Physics

Rondebosch 7701

South Africa

wheaton@hostess.phy.uct.ac.za

Yücel, Melike Behiye

Akdeniz University

Faculty of Sciences and Arts

Department of Physics

P.B.510, 07058, Antalya

Turkey

myucel@akdeniz.edu.t 